\title{
PATRIMONIO LOCAL QUE SE DEBILITA: LOS ARTESANOS DE GÓMEZ FARÍAS, JALISCO (MÉXICO)
}

Local heritage that weakens: the artisans of Gómez Farías, Jalisco (Mexico)

Jorge Arturo Martínez Ibarra Universidad de Guadalajara, México

jorge.martinez@cusur.udg.mx

Lizeth Espíritu Flores

Colectivo ATC Producción Audiovisual, México

ciateatrocusur@gmail.com

Rosa Elena Arellano Montoya

Universidad de Guadalajara, México

rosa.arrellano@cusur.udg.mx

José Jonathan Aguirre Zúñiga

Periodista independiente, México

latemtan@gmail.com

Recibido: 13-03-2018

Aprobado: 28-05-2018

Jorge Martínez lbarra es biólogo por la Universidad de Guadalajara, Maestro en Ciencias en Recursos Naturales y Desarrollo por El Colegio de la Frontera Norte y Doctor en Recursos Bióticos por la Universidad Autónoma de Querétaro, México. Es productor de radio y televisión documentalista, además de fundador del Colectivo Audiovisual En el camino andamos. Se desempeña como profesor-investigador el el Departamento de Artes y Humanidades del Centro Universitario Sur de la Universidad de Guadalajara, México.

\section{RESUMEN}

El presente trabajo surge como resultado de los documentales "Regalamos sonrisas, vendemos artesanías" (producido por Colectivo ATC-Producción Audiovisual) y "Una tradición que se debilita" (dirigido por Lizeth Espíritu y producido por el Programa de Apoyo a las Culturas Municipales y Comunitarias-Secretaría de Cultura del estado de Jalisco). A través de los testimonios de los artesanos del municipio de Gómez Farías (localizado en la zona sur del estado de Jalisco, en México), el documento presenta su sentir acerca de la labor artesanal, describiendo las características del proceso de colecta de las materias primas, la elaboración de las artesanías, los problemas a los que se enfrentan para la venta y sus reflexiones en torno a la permanencia generacional de esta actividad productiva.

Palabras clave: patrimonio local; artesanos; Gómez Farías 
Lizeth Espíritu Flores es egresada del Centro Universitario del Sur, de la Universidad de Guadalajara y colaboradora de la Compañía de Teatro del Centro Universitario del Sur, de la Universidad de Guadalajara. Pertenece al Colectivo ATC Producción Audiovisual de Gómez Farías, Jalisco.

Rosa Elena Arrellano Montoya es Licenciada en Contaduría Pública por la Universidad del Valle de Atemajac (UNIVA), Maestra en Ciencias en Recursos Naturales y Desarrollo Rural con orientación en Desarrollo Rural Productivo por el ECOSUR-SCLC, y Doctora en Ciencias Sociales con especialidad en Antropología Social por el CIESAS, SEP-CONACYT. Es es profesora de tiempo completo e investigadora en el Departamento de Artes y Humanidades del Centro Universitario del Sur de la Universidad de Guadalajara; es, también, responsable del Cuerpo Académico UDEG-CA-CulturArte.

José Jonathan Aguirre Zúñiga es periodista. Se desempeña en el área cultural.

\section{ABSTRACT}

This work arises is a result of the documentaries We give away smiles, sell handicrafts (produced by ATC-production Audiovisual collective) and "A weaken tradition " (directed by Lizeth Spirit and produced by Programme of Support for Municipal and Community Culture and the Ministry of Culture of Jalisco State). Through the testimonies of the artisans of the municipality of Gómez Farías, Mexico, this paper show his feeling about the artisan work, describe the collect of materials, the handicrafts elaboration process, the problems for sale and their reflections on the generational permanence of this productive activity.

Keywords: local heritage; craftmmen; Gómez Farías

\section{Introducción}

El patrimonio cultural de las comunidades locales se constituye de todos los bienes y valores culturales que expresan la identidad de un pueblo: bienes muebles e inmuebles, costumbres, hábitos, manifestaciones, representaciones y productos de la cultura popular (Gutiérrez y Gerritsen, 2011).

El entendimiento reciente del patrimonio cultural incluye no solo los monumentos y manifestaciones del pasado, sino también lo que se llama patrimonio vivo: las diversas manifestaciones de la cultura popular, las poblaciones o comunidades regionales, las lenguas indígenas, las artesanías y las artes populares, la indumentaria, los conocimientos, los valores, las costumbres y las tradiciones características de un grupo o una cultura. Por ello, el patrimonio cultural es un importante tesoro para las comunidades, como elemento básico de estabilidad social y referente de su identidad, y como por representante de un valor añadido de un territorio (Casasola, 1998). 


\section{La artesanía como parte del patrimonio cultural}

La artesanía es el conjunto de actividades que se hacen con las manos; por lo tanto, en su elaboración no se usan medios mecánicos (ningún tipo de máquina), pero sí herramientas que facilitan el trabajo. Antiguamente, cuando no existían las máquinas, todos los objetos se realizaban de manera artesanal; por eso la artesanía no se consideraba ni se considera un arte. Desde un punto de vista tecnológico, el producto artesanal se define a través de un proceso de producción fundamentalmente manual; desde una perspectiva antropológica, es el modo de producción tradicional por ser la tradición la que proporciona las técnicas, los útiles y los diseños; desde una óptica cultural, se funde con el concepto de arte popular, arte anónimo transmitido de padres a hijos (De la Calle, 2002).

Sin embargo, también existen expresiones artesanales que han incorporado al proceso productivo nuevas técnicas, materiales y diseños; así emerge un nuevo concepto de artesanía que engloba todas aquellas actividades que conservan un carácter diferencial, respecto a la producción industrial seriada. Como objeto patrimonial, presenta una ventaja indudable sobre otros: se puede transportar $y$, por lo tanto, sirve de remembranza y demostración de que se ha estado en un lugar. Además, hoy en día, rodeados como estamos de tecnología, se da un valor especial a los objetos artesanales, pues se aprecia el tiempo y la paciencia que los artesanos emplean en su elaboración (Rivera et al, 2008).

Las artesanías están asociadas con la economía campesina, ya que surgieron como un satisfactor de necesidades que ha pasado a ser objeto turístico por su rusticidad, colorido y diversidad. Esta actividad ha formado parte del trabajo cotidiano para muchas mujeres, hombres y familias completas que obtienen recursos a través de la elaboración de piezas. La artesanía tiene un valor intrínseco relacionado con la identidad del grupo social en el cual se realizan las piezas. Por ello el valor cultural se asocia al valor económico (Rivera et al, 2008).

\section{Los artesanos de Gómez Farías, Jalisco, México}

El sur de Jalisco está conformado por un medio natural diverso en donde históricamente se han desenvuelto una multiplicidad de actividades rurales y además, es considerada como una de las zonas con mayor riqueza cultural, 
manifestada en expresiones como la literatura, la pintura, la música, la gastronomía, la artesanía, etc. (Vargas, 2010).

No obstante, al igual que en el resto de México, el sector agropecuario en esta región atraviesa por una profunda crisis originada por una estrategia de desarrollo dirigida hacia industrialización de los espacios rurales: el establecimiento de agroempresas tomateras ajenas a la región, la creación de invernaderos para cultivos protegidos de exportación (arándano, zarzamora) con capital extranjero y el incremento considerable en la superficie cultivada de aguacate, han impactado negativamente todas las dimensiones del desarrollo (sociales, ambientales, económicas, culturales, políticas y éticas; OIEDRUSJALISCO, 2012; Vargas; 2013).

En contraparte, están los actores locales que mantienen una amplia diversidad de actividades productivas de larga tradición a menor escala como los equipales, la cajeta, la pitaya, la talabartería, el mezcal o la producción de artesanías. Todas estas actividades juegan un papel importante en la identidad regional, así como en la economía familiar y la integración regional, a partir de donde se pueden rescatar elementos para lograr la sustentabilidad rural y el desarrollo local (Vargas, 2013).

Parte de esta estrategia desde hace años la han desarrollado los artesanos de Gómez Farías.

"...Yo soy artesano por naturaleza y por nacimiento. No me imagino haciendo otra cosa en mi vida, pues ésta es la actividad que me mantiene pleno, lo que mejor se hacer y lo que he hecho la mayor parte de mi vida...".

José Reyes Flores Gaspar, artesano (62 años)

"...Yo me enseñé gracias a mis padres, desde que tenía como 10 años, todos mis hermanos trabajan haciendo esto, mis hijas también lo hacen, y ahora sus hijos están aprendiendo y ya nos ayudan en lo que pueden; sí, es una tradición ser artesano..."

Ma. de Jesús Sixto, artesana (72 años)

El proceso de creación de un artículo artesanal elaborado en Gómez Farías está basado en tres etapas: la recolecta de la materia prima (otate, tule y carrizo), la elaboración de las artesanías y la comercialización de éstas. Si bien no existe un archivo oficial que certifique el origen del tejido de estas materias primas, dentro de la memoria histórica oral se atribuye el comienzo de éste trabajo artesanal a los frailes franciscanos durante la evangelización del pueblo. 
"...Ya son fechas muy antiguas y no me di cuenta yo de eso. Quién sabe quiénes serían los primeros que empezaron con esto del otate y el carrizo. Yo me imagino que desde que se fundó aquí, creo que empezó con personas que trabajaron petates, sillas, canastas, ollas...".

\section{Emilio Venancio, artesano (79 años)}

"...Vinieron a instruirnos unos frailes de la congregación de Vasco de Quiroga, por eso es que nosotros tenemos mucha hermandad con los productos. Porque se topan los frailes que tenemos el mismo clima y la misma vegetación, tenemos una laguna como ellos la tienen. Ellos nos enseñaron a trabajar, nos enseñaron a tejer sillas... Yo creo que hace un buen rato, unos ciento setenta años, porque mis abuelos ya sabían..."

"...Antes de que ellos vinieran (los frailes franciscanos), se hacía un tejido con izote y no era tejido, sino puros nudos amarrados. Últimamente hemos recibido cursos, ese mismo tejido duró muchos años. Posteriormente fue éste que se llama de gajos. Ya últimamente otros que son diferentes, más evolucionados...".

Ma. Eduviges Martínez Benito, artesana (52 años)

\section{a) Recolecta de materia prima}

El otate (Otatea acuminata) solo puede encontrarse en cerros y barrancas aledañas a Gómez Farías. Los recolectores recorren entre dos y tres horas a caballo para llegar hasta donde se encuentra la planta. Para el proceso de corte en el campo (en el cual se invierten aproximadamente dos horas) se requieren guantes para no lastimarse las manos, una casanga para cortar las varas, un trozo de soga para amarrar la carga a la mula o caballo de carga, gorra y sudadera para protegerse del sol (Espíritu et al, 2013).

La selección de las varas depende del pedido que le hayan solicitado al recolector, si el artesano quiere realizar canasta grande, su encargo será conseguir otate grueso y largo, que no esté "picado" por los gusanos, que no esté seco o sucio pues el material debe estar en buen estado (Espíritu et al, 2013).

"...Con una carga yo gano al rededor de $\$ 200.00$ (US $\$ 11.7$ ) pero hay veces que me llevo más, ahí me puedo ganar $\$ 300.00$ (US \$17.6); a mí no me regatean porque la gente sabe que el material que le llevo no es material corriente..."

Cecilio de la Cruz, recolector de otate (56 años) 
Fotografía N 1. Cecilio de la Cruz (56 años), recolector de otate.

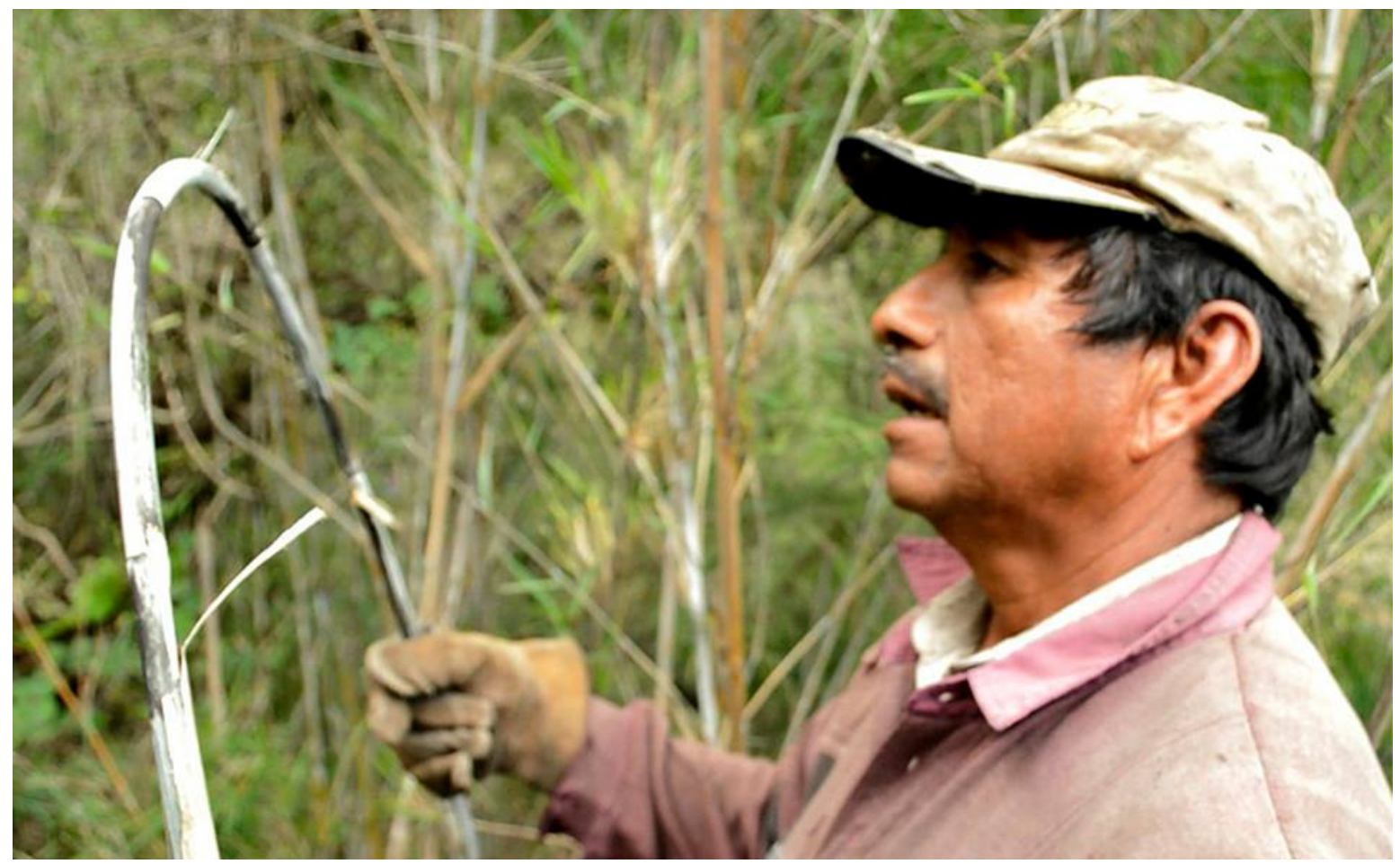

Fotografía: Lizeth Espíritu.

El tule (Typha spp) se obtiene en la Laguna de Zapotlán, localizada al suroeste de la cabecera municipal. Los recolectores utilizan un tapeixte (lancha elaborada totalmente de tule con ayuda de alambre) o una lancha convencional (frecuentemente prestada por los pescadores del sitio). Utilizando una vara larga y gruesa para impulsar la embarcación en las aguas poco profundas de la laguna; transita entre la vegetación acuática donde selecciona el área más cómoda para trabajar. Se toman como referencia los siguientes criterios para su extracción (Jiménez, 2012):

- Tamaño de la planta

- Consistencia

- Color

- Textura 
Para cortar el tule utiliza una guadaña o machete y comienza a cortar donde la planta deja de tocar el agua y sale a la superficie del lago. Se busca tule que no esté manchado y tenga muchos usos. Una vez cortado, lo sube al bote y continúa cortando, hasta llenar la embarcación. Cuando ya se tiene el suficiente se acerca a la orilla, se deposita el tule sobre la tierra y se pone a secar. Posteriormente, se encarga de distribuir los pedidos a los artesanos que se lo solicitaron (Espíritu et al, 2013). La manotada de tule la ofrece en $\$ 20.00$ (US\$1.1) y el tercio en $\$ 100.00$ (US\$ 5.8; ambas cantidades establecidas coloquialmente y reconocidas entre los artesanos y el recolector).

Los cortadores de tule señalan que entre otros problemas, no cuentan con un respaldo de ninguna dependencia del gobierno mediante la expedición de permisos para el corte y explotación del tule, ningún apoyo en créditos para la creación de infraestructura que facilite la realización de sus actividades y eleve su productividad, como por ejemplo, la adquisición de lanchas que faciliten el corte del tule o el acondicionamiento de terrenos aledaños a la laguna para facilitar el secado del tule cortado (patios), lo cual siempre les representa problemas cuando está verde (Jiménez, 2010).

La mayor parte de la mano de obra que se emplea en el corte de tule es permanente y su oficio fue heredado de las generaciones anteriores. Sin embargo, anteriormente el corte del tule era una actividad que generaba más empleo; se formaban grupos y llegaron a tener una asociación de cortadores. Hoy, los actuales cortadores lo hacen por temporadas, es decir, cuando no encuentran otro empleo mejor remunerado (Jiménez, 2010).

Muchos cortadores proveen de la materia prima a sus propios familiares quienes se encargan de la elaboración de productos artesanales, ampliándose con esto los beneficios económicos con la agregación de valor. En otros casos se realizan prácticas de "medieros" entre los cortadores y los artesanos, es decir, después de la venta de los productos, se reparten los beneficios entre los dos en la misma proporción (Jiménez, 2010).

"...A mí me gusta lo que hago, son muchos años de cortar tule y le agarra uno el gusto, aunque terminamos todos mugrosos, eso no nos quita que sigamos en esto...".

José Nolasco, recolector de tule (67 años) 
Fotografía N² 2. José Nolasco (67 años), recolector de tule.

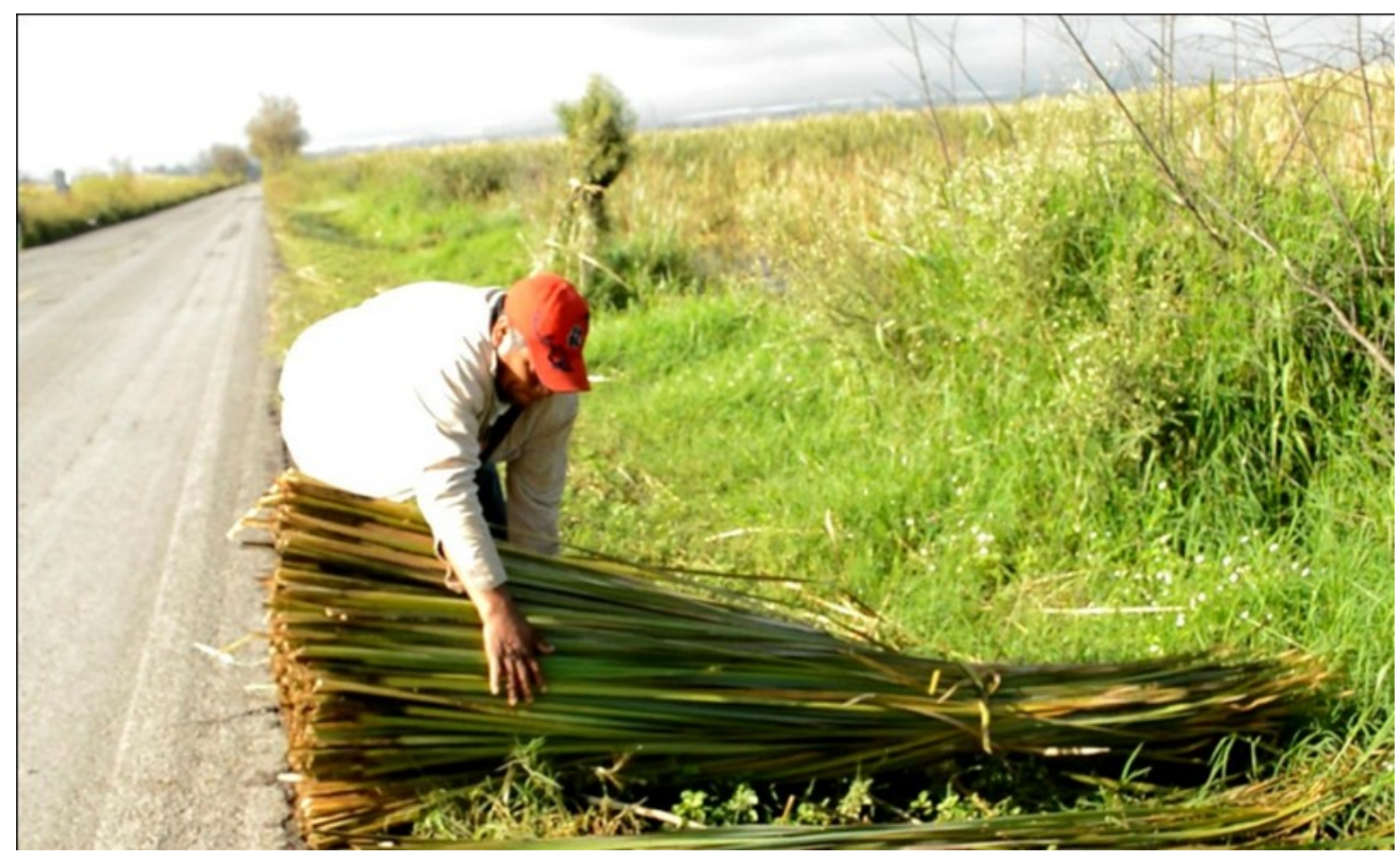

Fotografía: Lizeth Espíritu.

El carrizo (una especie de gramínea), es la tercera planta utilizada como insumo de las artesanías de Gómez Farías, por lo general se encuentra en los corrales (traspatios) o en sitios de fácil acceso aledaños a la comunidad.

\section{b) Elaboración de artesanías}

Una vez que el proveedor hace la entrega del material a los artesanos, éstos seleccionan, separan y clasifican el material dependiendo del artículo que se vaya a elaborar: el tule ancho lo utilizan por lo general para tejer sillas, sopladores o tortilleros, en cambio el que se encuentra algo rollizo lo ocupan para tejer petate. Usualmente en la elaboración de artesanías de tule se utilizan moldes de madera que sirven como base para formar canastas, bolsas o sombreros (Espíritu et al, 2013).

La confección de artesanías de tule requiere que el material tenga un secado de al menos dos semanas; de hecho, este material puede durar resguardado hasta un año, siempre y cuando se tengan los cuidados necesarios para que no se 
maltrate, se apolille, se manche o se pudra. El otate nuevo se utiliza para la base de las canastas y el viejo funciona para darle cuerpo a éstas. Las herramientas que se utilizan son: cuchillo, mazo y anillos de hule que sirven para no astillarse al momento de darle forma al producto (Espíritu et al, 2013).

“...Para hacer el diseño de una canasta se deben cortar los trozos por mitad, y machacar esto para evitar que el carrizo se raje. Luego, se aplasta con el mazo y apoyo de una piedra; con ayuda del cuchillo se lija por dentro para que quede liso el asiento de la canasta, se quitan imperfecciones y se marca con un lápiz la medida deseada, para continuar con trozos más delgados de carrizo y así lograr la figura solicitada. Las varas se tienen que rajar para que el carrizo no nos astille, y la correa quede derechita, así el trabajo estará bien hecho..."

Juan Venancio, artesano de carrizo (78 años)

Fotografía № 3. Juan Venancio (78 años), artesano de carrizo.

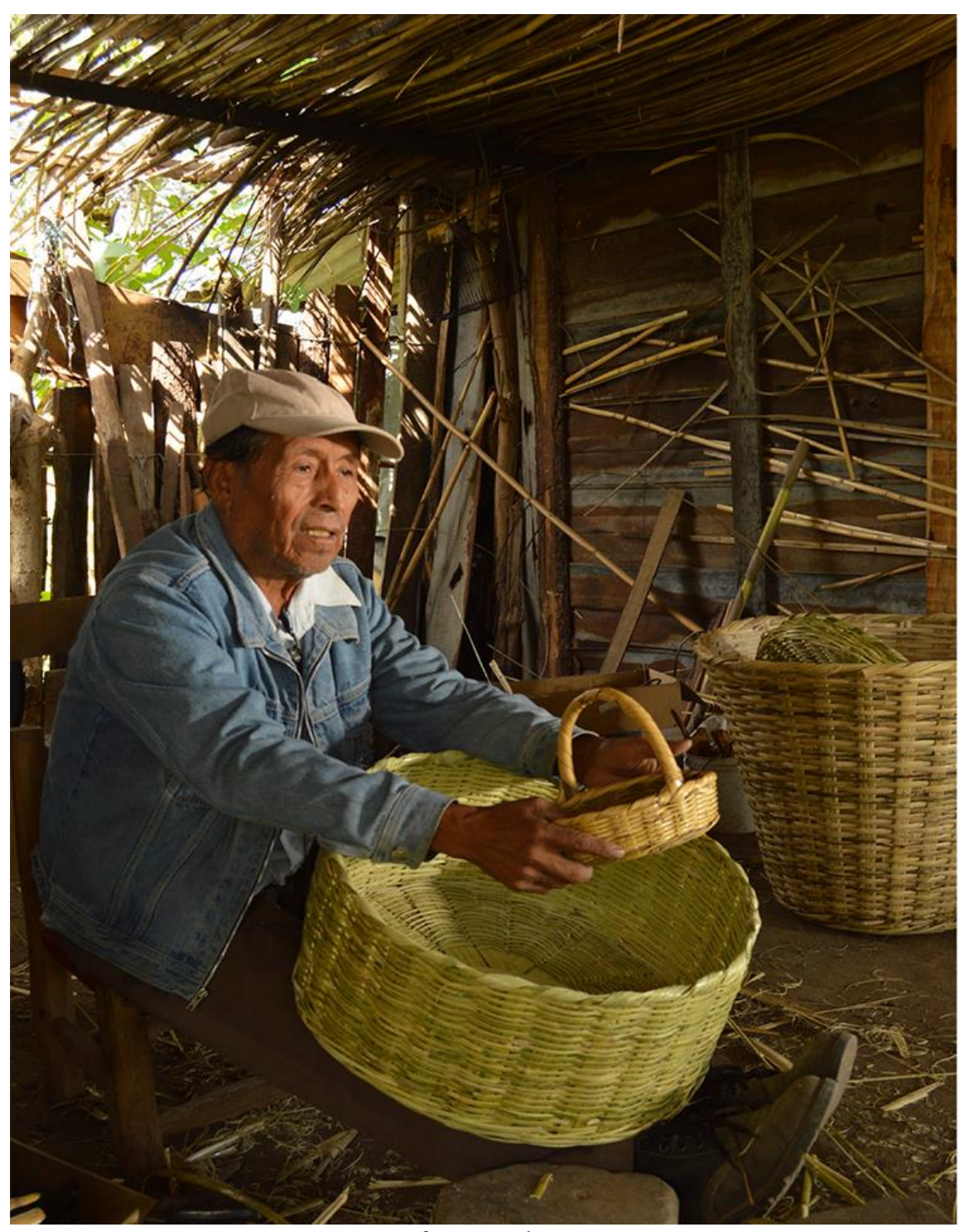

Fotografía: Lizeth Espíritu. 
Fotografía № 4. Entrelazado del tule para la elaboración de canastas.

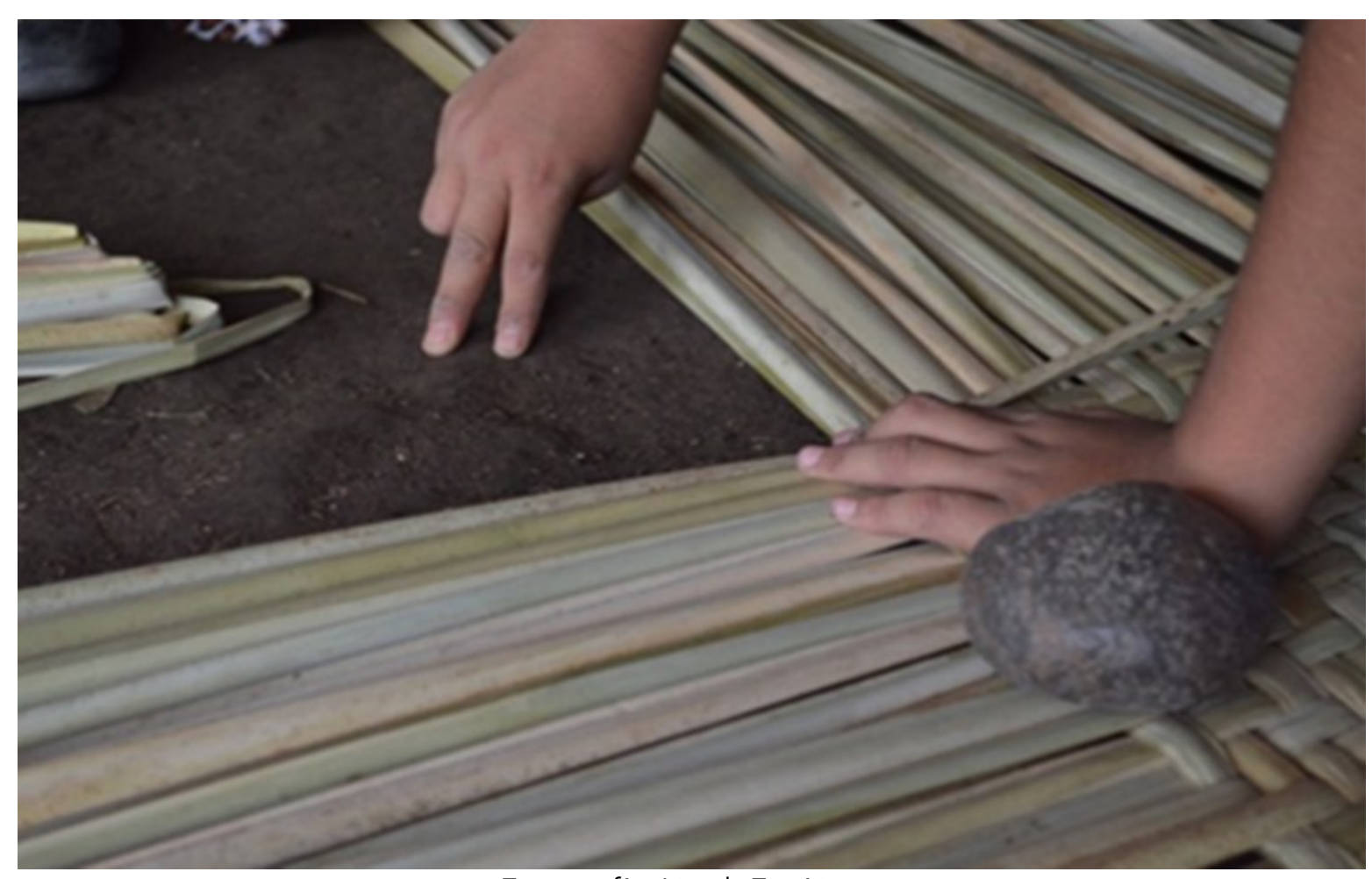

Fotografía: Lizeth Espíritu.

\section{c) Comercialización}

La mayoría de los artesanos del municipio que se dedica a esta actividad se encuentran organizados como microempresas familiares. De ellos, algunos tienen sus talleres y puntos de venta en sus propios domicilios particulares; otros prefieren intentar la venta fuera de su municipio, principalmente durante las festividades patronales de la región y la tercera opción es la venta en el Tianguis Artesanal, un espacio de exhibición y venta localizado a un costado de la carretera libre Guadalajara-Colima km 82.

No obstante, los artesanos se enfrentan a diversos problemas durante el proceso de comercialización de las artesanías, como son: la carencia de espacios adecuados y suficientes para la venta (las instalaciones del Tianguis Artesanal son pequeñas e insuficientes para albergar a todos los artesanos); la falta de recursos económicos, vehículos, infraestructura y canales de comercialización para distribuir las artesanías fuera de la cabecera municipal; la demanda de 
grandes empresas, restaurantes, cadenas hoteleras o particulares de la producción de grandes volúmenes en poco tiempo (lo cual dado que son productos artesanales, lo hace totalmente inviable); la imposibilidad de expedir facturas o recibos fiscales; el desconocimiento de la forma de acceder a nuevos mercados mediante, por ejemplo, las tecnologías de la información; y; sobre todo, el regateo (solicitud del cliente de disminuir el precio del producto, Espíritu et al 2013; Vargas 2005, 2013).

“...Las personas piensan que porque es un material que está en la laguna, el producto debe ser muy barato, pero en sí no es el tule lo que cuesta sino la transformación de la materia en un artículo..."

María de Jesús Ortiz, artesana (42 años)

Irónicamente, existen intermediarios que han logrado posicionar estas artesanías tanto en los mercados nacionales como exportarlos a algunas partes de Estados Unidos, Canadá, Puerto Rico, Inglaterra y Japón obteniendo con ello altas utilidades (Jiménez, 2012).

\section{d) La pérdida de la tradición artesanal}

El uso de artículos elaborados con materiales como plástico, metal, papel, cartón, etc., por parte de los consumidores, disminuyen paulatinamente el interés de éstos por adquirir artículos artesanales de uso cotidiano, además de que el costo de los primeros (elaborados en serie la mayoría de las veces, lo que disminuye ampliamente sus costos de producción) es mucho más económico. Ello implica que los productos artesanales de Gómez Farías se perciban cada vez más como artículos decorativos, más que utilitarios.

Finalmente, las nuevas generaciones del municipio se muestran poco interesadas en continuar la tradición artesanal, principalmente debido a la dificultad cada vez mayor para conseguir materia prima de buena calidad, a lo complicado que resulta el proceso de producción, a la incertidumbre en la comercialización (y por lo tanto, en la generación de ingresos económicos), al bajo precio otorgado por los compradores y a la carencia de apoyos institucionales.

"...Si no hay capacitaciones, talleres, cursos, para los adolescentes, jóvenes, mujeres del municipio ésta tradición se va a perder... pero también no se interesan, a mí me invitaron una vez a dar un taller a cincuenta mujeres... en tres meses les enseñé a trabajar con tule, para que supieran hacer bolsas, petatitos, sopladores, canastas... 
pero ahorita dígame, quiénes lo trabajan, ninguna, también eso no está bien, no se interesan, y pues esto se va a perder en unos años..."

Miguel Hernández, artesano (62 años)

"...Mi hijo me ayudó un tiempo, me veía que me apasionaba por este trabajo y que tenía algo de clientes... se enseñó, pero después, dejó de hacerlo porque las ventas bajaron y la gente quería todo más barato... él se desesperó y mejor dijo que iba a estudiar, ya no se acerca a apoyarme en esto de la artesanía, reniega de que la gente no pague bien y pues, es un trabajo que tiene su dedicación, como todo...".

José Reyes, artesano, (54 años)

“...Yo tuve 2 hijos hombres y cuando eran chamaquitos sí me ayudaban aquí a hacer petates, canastas... ahorita de mis hijos ninguno siguió con esto que hago yo, uno trabaja en el campo y le va bien, sale para sus gastos, a mí ya me ajusta para frijoles y tortillas..."

Emilio Venancio, artesano, (79 años) 


\section{BIBLIOGRAFIA}

Casasola, Luis. (1998). Turismo y ambiente. México: Editorial Trillas Turismo.

Espíritu, Lizeth et al. (2013). Regalamos sonrisas, vendemos artesanías. Documental sobre familias artesanas de Gómez Farías, Jalisco. México: ATC Producción Audiovisual, Universidad de Guadalajara.

Gutiérrez Estrada Marisa y Peter Gerritsen. (2011). Turismo rural sustentable. Estudio de caso de la Costa Sur de Jalisco. México: Plaza y Valdéz Editores/Centro Universitario de la Costa Sur, Universidad de Guadalajara.

INESER-CUCEA-UDG. (2000). Monografía del municipio de Gómez Farías, Jalisco. México: Centro Universitario de Ciencias Exactas e Ingeniería (CUCEA), Departamento de Estudios Regionales, Universidad de Guadalajara.

Jiménez Mejía, Francisco de Paul. (2012). Plan Municipal de Desarrollo de Gómez Farías, Jal. 2012-2015. México: H. Ayuntamiento Constitucional de Gómez Farías, Jal. 2012-2015.

OEIDRUS-Jalisco. (2012). Anuarios estadísticos. SAGARPA. Dirección: http://www.oeidrusjalisco.gob.mx/agricultura/anuarios/index.php?idcurrent=2 \&nivel=MUN. Consulta: 16 de febrero de 2018.

Rivera Cruz, María Leticia et al. (2008). La artesanía como producción cultural. México. Convergencia, Revista de Ciencias Sociales. UAEMex, núm. 46, eneroabril. Pp. 225-247.

Vargas Hernández, José. (2005). Capital social organizacional base de la sustentabilidad organizacional para el desarrollo ambiental y económico: el caso de las micro y pequeñas empresas en la explotación del tule Typha spp de la Laguna de Zapotlán. Colombia. Revista CIFE, Vol. 12, No. 16. Pp. 155-173.

Vargas Hernández, José. (2010). Sustentabilidad organizacional para el desarrollo ambiental y económico. El caso de micro empresas en San Sebastián del Sur. Venezuela. Revista Venezolana de Análisis de Coyuntura, 2010, Vol. XVI, No. 1 (ene-jun). Pp. 187-210. 
Vargas Hernández, José. 2013. Desarrollo regional y sustentabilidad: turismo cultural en la región sur de Jalisco. Brasil. Cad. Metrop., São Paulo, v. 15, n. 29 (jan/jun). Pp. 313-338.

\section{ENTREVISTAS}

Cecilio de la Cruz, recolector materia prima, Gómez Farías, noviembre 1, 2013.

José Nolasco, recolector materia prima, Gómez Farías, octubre 31, 2013.

José Reyes Flores, artesano Gómez Farías, octubre 28, 2013.

María de Jesús Sixto, artesana Gómez Farías, octubre 24, 2013.

Juan Venancio, artesano Gómez Farías, octubre 10, 2013.

María de Jesús Ortiz, artesana Gómez Farías, octubre 31, 2013.

Ma. Eduviges Martínez Benito, artesana Gómez Farías, octubre 10, 2013.

Rafaela Venancio, artesana Gómez Farías, octubre 28, 2013. 